\title{
The Acoustic Emission Signal Recognition based on Wavelet Transform and RBF Neural Network
}

\author{
Shaohui Ma and Xiangqian Chen \\ Henan Mechanical and Electrical Engineering College, Xinxiang 453002, China \\ hgzhou2002@sina.com
}

\begin{abstract}
The acoustic emission (AE) technology can be used to assess the security condition of oil storage tank without opening pot. Signal recognition is a foundation to analyze the corrosion status for oil storage tanks. Because of inadequateness of the analysis method of parameters, a new acoustic emission signal recognition method is proposed based on wavelet transform and RBF neural network. AE signal was decomposed to 6 layers by db2 wavelet and the space energy of 6-layer detail features is regarded as the vector of the AE signal characteristics. $R B F$ neural network is designed by considering the characteristics of AE signal. The RBF neural network is trained by using the pattern known of acoustic emission signal. RBF network is used to classify experiments to corrosion, crack and condensation acoustic emission signal. The experimental results show that the recognition rate of RBF neural network reaches $93.3 \%$, which reveals the advantage of the acoustic emission signal of neural network recognition. It has some significance of the quantitative analysis to the safety situation of oil storage tanks.
\end{abstract}

Keywords: AE signal, wavelet decomposition, feature extractio, RBF neural network, recognition

\section{Introduction}

AE signal is weak from the tank floor corrosion, which is affected by environment and distance in the sampling process containing certain noise. How to extract characteristics of corrosion type from AE signal containing noise [1] is to conduct an effective analysis for the feature extraction, which becomes a key problem. It is important for the storage tank safety situation to do the quantitative analysis. Early analysis of AE signals adopted parameter analysis method. The typical acoustic emission signal parameters include amplitude, ring down count, duration, energy, threshold voltage, time of arrival, hit number rate [2]. With the further application research of AE technology, some new analysis methods of parameters are proposed and used. Lim, et al., proposed to use the ratio of signal peak and a time signal average value, that is to say, the Crest parameter to analyze characteristics of pipeline leakage AE signal [3]. The domestic scholar, Ma Yonghui used energy, duration, amplitude, count and other conventional parameters to analyze the relation between neural network and grey correlation, and successfully distinguished several classes of the AE signal of sound metal pressure vessel [4]. Although the parameter analysis method is simple and easy to understand and measure, there are also some problems that the choice of the parameter has the large arbitrariness, and $\mathrm{AE}$ signal parameters are to just describe one or some features of the wave form of the AE signal, which characterizes the limitations of the entire AE signal source. Wavelet analysis has the good signal noise separation and time-frequency localization characteristics, which is especially suitable for the feature extraction of the AE signal. Suzuki, et al., used fast Fourier transform; short-time Fourier transform and wavelet transform analysis methods respectively, The analytical results show that the wavelet analysis can extract more $\mathrm{AE}$ source characteristic information [5]. 
Gang, et al., carried out discrete wavelet decomposition to the AE signal of carbon fiber composite materials to study its properties [6]. Cui Yan, et al., used the wavelet analysis to study the $\mathrm{AE}$ signal characteristics of discontinuous reinforced metal composite material interface [7]. In the analysis aspects of the classification on characteristic of AE signals, because of artificial neural network imitating the brain neural network, it can effectively deal with the non-linear, fuzzy and uncertainty relations of the problem, with the very strong adaptive, self-organizing ability, which is widely used [8]. In 1989, Wsachse, et al., began to study the AE signal processing by the artificial neural network [9]. Baily and Mathew used the neural network to develop the model which is used for diagnosis to the defects of balls [10]. Based on the neural network, Tadej and Kosel invented the intelligent detection source of the AE [11]. Zhao Yuanxi used the wavelet package to decompose acoustic emission signal of rolling bearing with different faults to distinguish the fault type of rolling bearing by the neural network [12].

Because the AE signal is interfered susceptibly by some noise, and different AE signal are mainly reflected in the specific features [13], the wavelet analysis has a unique advantage in denoising and extracting the detail features. The RBF neural network can approximate any nonlinear function, compared to the other neural network such as BP network [14], which can handle the difficult analytic regularity of the system inherent. As it approximates locally the network, it has the advantages of the fast convergence of the learning speed to meet the characteristics of $\mathrm{AE}$ signal. The wavelet analysis is used to extract detail features of 6 layers of AE signal. The space energy of each detail feature is regarded as the characteristics of the $\mathrm{AE}$ signal. According to the characteristics of $\mathrm{AE}$ signals to design RBF neural network to test the classification performance to the $\mathrm{AE}$ signal. The experimental results show that the extracted features can distinguish well the $\mathrm{AE}$ signal of corrosion, crack and condensation, and the RBF neural network design has the small error, and therefore the correct recognition rate is up to $93.3 \%$, which shows the superiority of RBF neural network recognition to the AE signal.

\section{The AE Signal Feature Extraction based on Wavelet Transform}

\subsection{The Theory of Wavelet Transform}

The breakthrough of the discrete wavelet transform is Mallat algorithm, a kind of fast algorithm proposed by S. Mallat in 1989 based on the multi resolution analysis [15]. The high passes filter Hi_D and the low pass filter Lo_D in time domain are used to decompose the signal. The first layer decomposition is taken as an example. The decomposition signal is assumed as $S$, which is in the approximate part of the first layer. The $c A_{1}$ of the wavelet coefficients in the low-frequency part of is through the signal $S$ and the convolution of Lo_D low pass filter, and then the result of the convolution is attained by alternately sampling. In the second layer, $S$ is replaced by $c A_{1}$. The same method is used for the decomposition. In the $j$ layer, the signal $f(t)$ is decomposed into $c A_{j}$, wavelet coefficients of approximation part and $c D_{j}$, wavelet coefficient of detail part, which is shown in Figure 1.

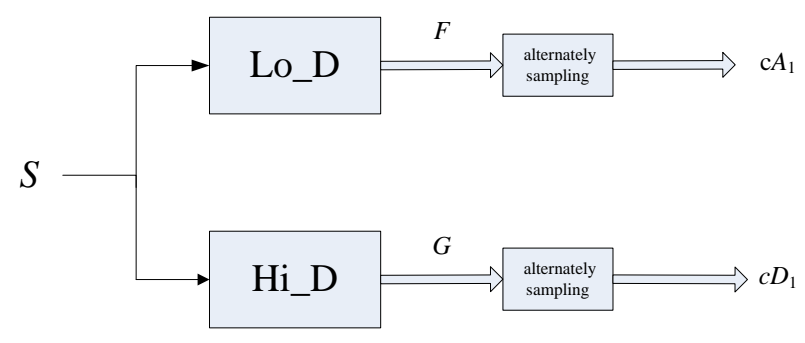

Figure 1. One-dimensional Discrete Wavelet Decomposition Transform 


\subsection{The Feature Extraction of AE signal}

$5 \%$ diluted hydrochloric acid is used, and $2.5 \% \mathrm{NaCl}$ solution is used to simulate acoustic emission signal of electrochemical corrosion to the metal plate, as shown in Figure 2. Nielsen-Hsu [16] Broken lead method is used to simulate acoustic emission signals of metal plate crack, as shown in Figure 3. The drip experiment is used to simulate the acoustic emission signal of condensation as interference signal, at dripping speed about 1Hit/s, as shown in Figure 4. The sampling frame length is 8192, and the sampling frequency is $2 \mathrm{MHz}$. Before the wavelet decomposition, the denoise method in the literature [17] is used to denoise the acoustic emission signal. db2 is selected to decompose the wavelet base as shown in Figure 5. The one-dimensional acoustic emission signal is decomposed into six layers, and get an approximate characteristic coefficient $c A_{6}$ and the 6 detail characteristic coefficient $c D_{j}, \quad(\mathrm{j}=1,2, \ldots, 6)$. In Figure 6 , the decomposition of AE signal of the condensation is taken as an example.

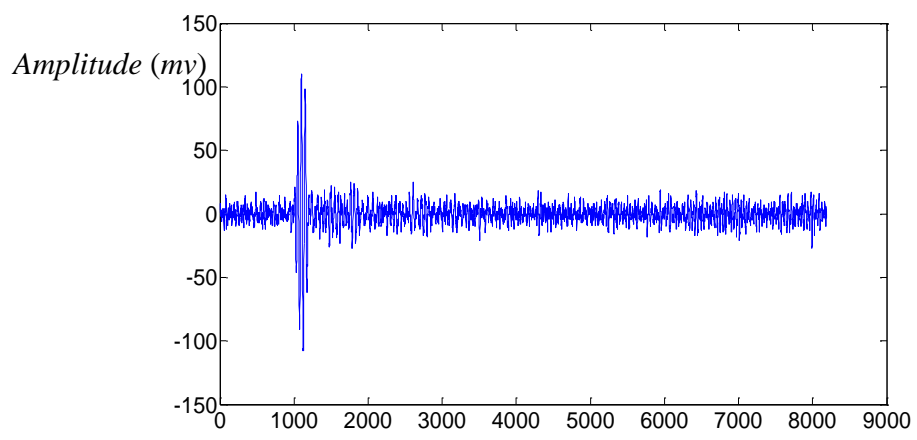

sampling number

Figure 2. Corrosion AE Signal

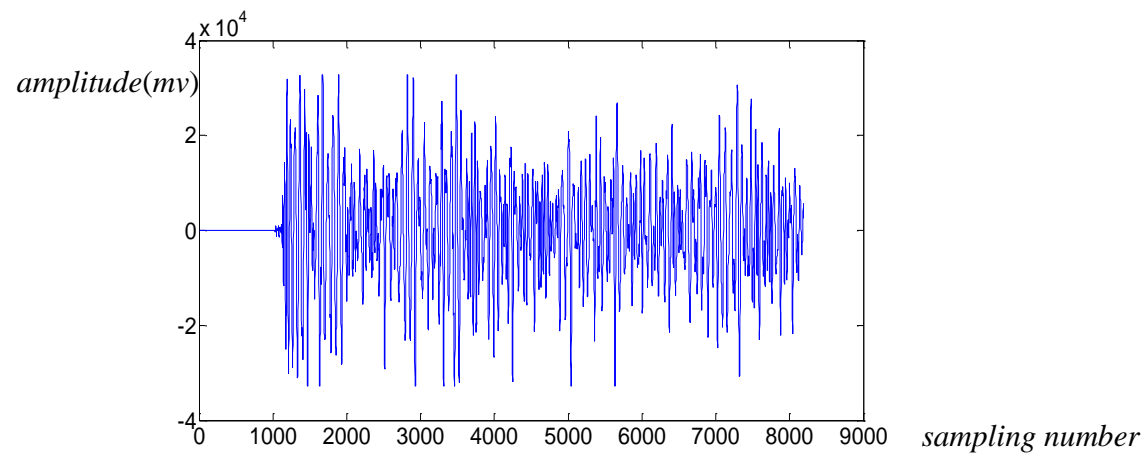

Figure 3. Crack AE Signal

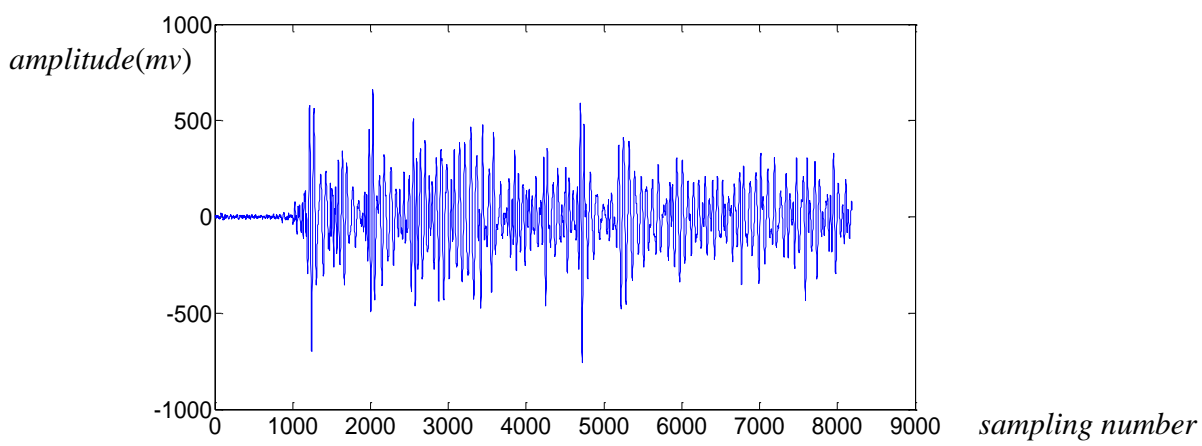

Figure 4. Condensation AE Signal 


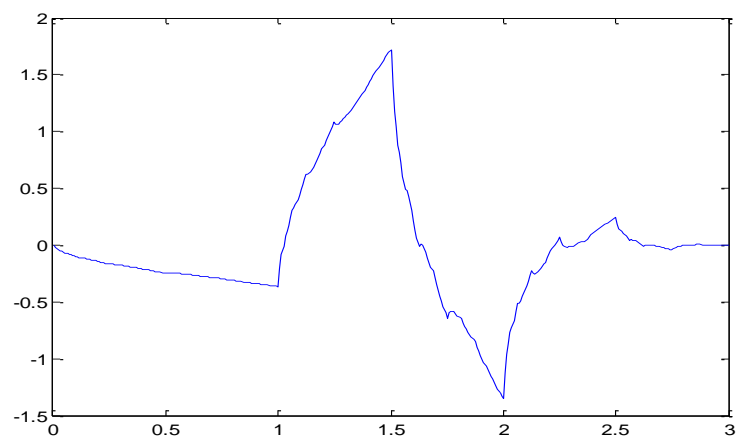

Figure 5. Db2 Wavelet Filter

Because the detail feature is an important index to distinguish between different types of AE signals, all the details characteristic coefficients of AE singal is used for the feature extraction. The coefficients of detail feature are huge in quantity, and therefore in order to facilitate the identification AE siganal by neural network, the energy of the 6 spaces of the detail features are calculated and are normalized as the vector $\mathrm{X}$ representative of the $\mathrm{AE}$ signal.
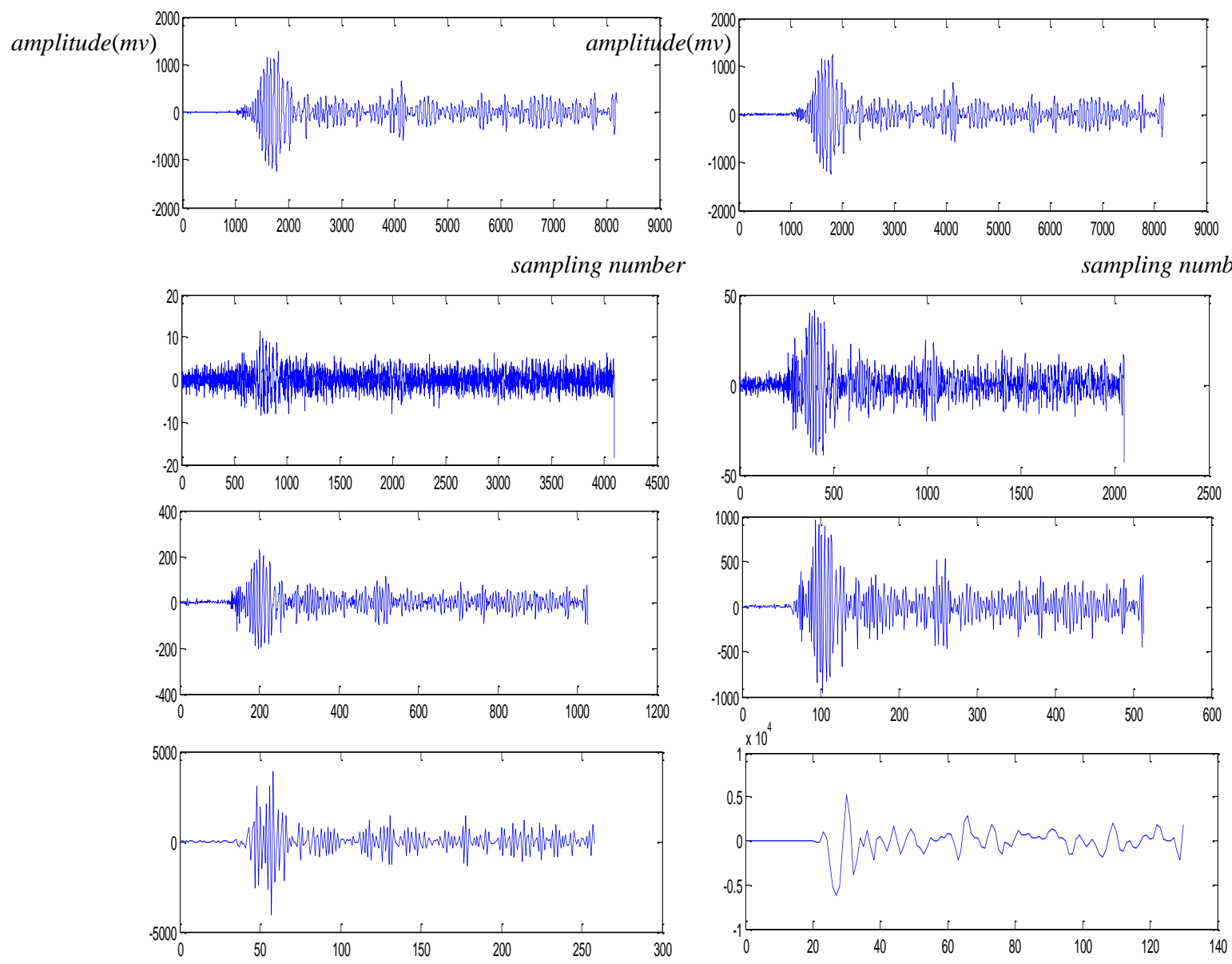

Figure 6. Detail Characteristic Coefficient of Condensation AE Signal by 6-layers Wavelet Decomposition 


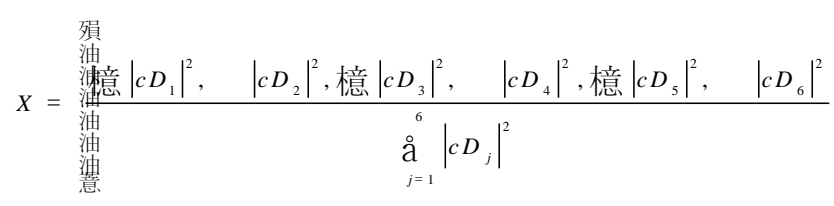

In Figure 7, the energy distribution is shown, which is of the space of each detail feature of three kinds of AE signals after the6 layer decomposition of db2 wavelet. The transverse coordinate $i \quad(i=1,2,3,4,5,6)$ represents the detail feature space of the $i$ layer.

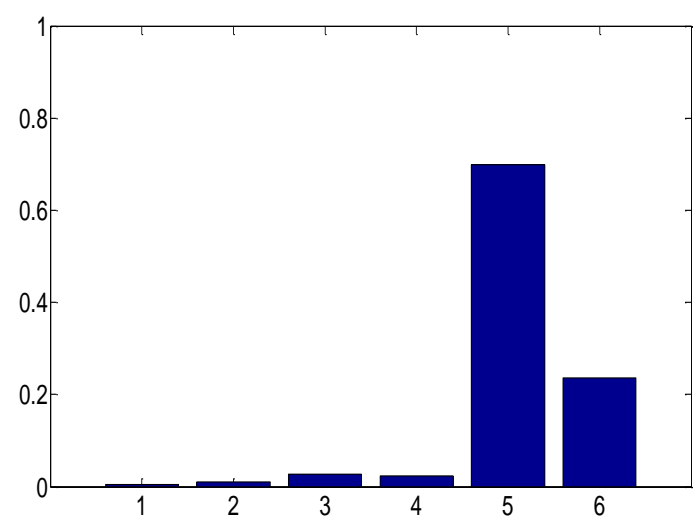

(a) Corrosion AE Signal

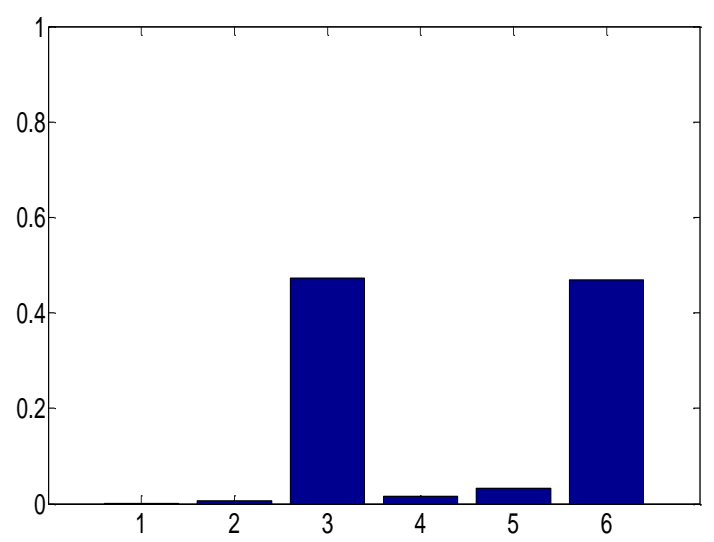

(b) Crack AE Signal

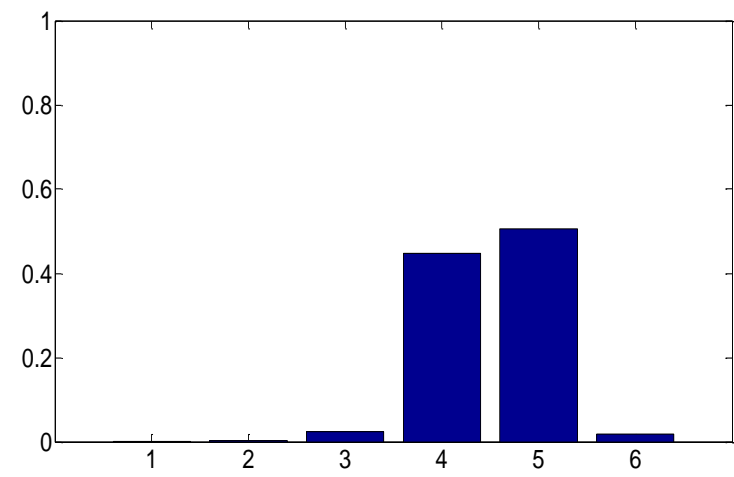

(c) Condensation AE Signal

Figure 7. Energy Distribution of Detail Feature Space 
By analyzing Figure 7, the space energy of the detail feature of the corrosion AE signal is mainly concentrated in the fifth and the sixth layers. The space energy of the detail feature of the condensation AE signal mainly is concentrated in the third and sixth layers. The space energy of the detail feature of the crack AE signal is mainly concentrated in the fourth and fifth layers. It can be seen that the extracted features distinguish obviously. Based on the wavelet decomposition, the characteristics of $\mathrm{AE}$ signals of all detail coefficients can distinguish various types of $\mathrm{AE}$ signal.

\section{The Structure of RBF Neural Network}

RBF neural network is three-layer feed forward neural network with the single hidden layer, whose biggest feature is the transfer function of hidden layer as a local response function. Compared with other multilayer feed forward neural network, it needs the greater number of hidden layer neurons, but requires less training time, and can approximate any continuous function [14] at any accuracy. In Figure 8, the general structure of the RBF neural network is shown. Network has $N$ input nodes, $P$ hidden nodes, and $l$ output nodes. The number of hidden nodes is usually selected for the number of input samples. The center of the radial basis function is regarded as the input samples. The radial basis function takes the expansion constant of uniform. Each layer in the mathematical description is as follows: $X=\left(x_{1}, x_{2}, \cdots, x_{N}\right)^{\mathrm{T}}$ is regarded as the input vector of the network. The hidden node activation function $G$ represents the Green function, Gauss function is generally chosen as shown in formula (2). $Y=\left(y_{1}, y_{2}, \cdots, y_{l}\right)^{\mathrm{T}}$ represents the network output. The output layer $\sum$ is the linear activation function.. The output layer uses 2 neurons to represent 3 kinds of signals. $(1,1)$ is the corrosion signal; $(0,1)$ is the condensing signal; $(1,0)$ is the crack signal.

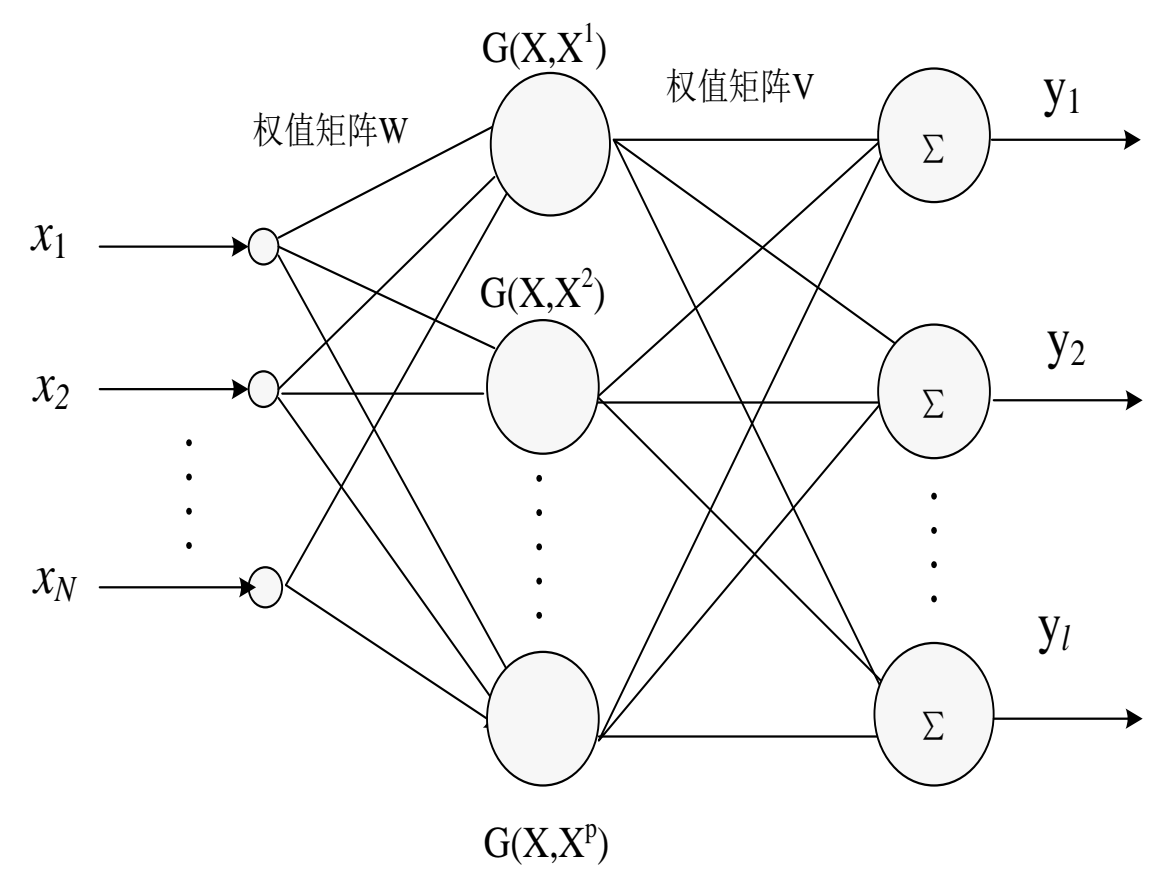

Figure 8. The Structure of RBF Neural Network

$$
\mathrm{G}\left(\mathrm{X}, \mathrm{X}^{\mathrm{p}}\right)=\mathrm{e}^{-\frac{1}{2 \delta_{p}^{2}}\left\|X-X^{p}\right\|^{2}}
$$




\section{The Results and Analysis of the Experiments}

The feature vectors extracted from 10 groups of corrosion AE signal are regarded as training input vector $X$, as shown in Table 1 . The target vector of AE signal is $O[1,1]$. The RBF neural network is trained by the $X$ and $O$, and then the RBF network trained is used to simulate the AE signal recognition. The initial hidden nodes of RBF neural network are set to the number of training samples. The based function selects Gauss function, and the data center is regarded as training samples themselves. The training mean square error is set to 0.001 . Because the number of training samples is not large, the extend constant is set to 1.2 [18]. The number range of hidden nodes is set to 7-13. Table 2 shows the error between the simulation results and the target vector $O$ in different number of neurons in hidden layers. When the number of neurons in hidden layer is 10 , the error between the actual network output and the expected output is the smallest. In Table 3, when the number of neurons in hidden layer is 10, the RBF network after the 108 steps training achieves the error of the objectives, and the number of steps is the smallest; the convergence speed is the fastest. Based on the above results, the number of neurons in hidden layer of the RBF network is set to 10 . Based on the above analysis, it can be seen that the network performance is not improved with the increase of the number of hidden layer neurons.

10 groups are extracted randomly from each type of $\mathrm{AE}$ signal to test the trained $\mathrm{RBF}$ network. 1-10 groups are AE signals of the corrosion; 11-20 groups are the condensation $\mathrm{AE}$ signal; 21-30 groups are the crack $\mathrm{AE}$ signal of. After the test, the correct recognition rate of RBF network is up to $93.3 \%$.

Table 1. Input Vector $X$ for Training

\begin{tabular}{cccccccccc}
\hline Group & Group & Group & Group & Group & Group & Group & Group & Group & Group \\
1 & 2 & 3 & 4 & 5 & 6 & 7 & 8 & 9 & 10 \\
\hline 0.0114 & 0.0106 & 0.0176 & 0.0227 & 0.0026 & 0.0127 & 0.0169 & 0.0179 & 0.0080 & 0.0076 \\
0.0186 & 0.0197 & 0.0302 & 0.0382 & 0.0068 & 0.0231 & 0.0291 & 0.0327 & 0.0149 & 0.0138 \\
0.0429 & 0.0435 & 0.0602 & 0.0727 & 0.0308 & 0.0477 & 0.0519 & 0.0567 & 0.0362 & 0.0374 \\
0.1873 & 0.1642 & 0.1799 & 0.1852 & 0.2663 & 0.1505 & 0.1497 & 0.1619 & 0.2059 & 0.1843 \\
0.6012 & 0.6401 & 0.5128 & 0.4861 & 0.5478 & 0.5553 & 0.4499 & 0.5156 & 0.5955 & 0.5880 \\
0.1386 & 0.1219 & 0.1993 & 0.1952 & 0.1456 & 0.2107 & 0.3025 & 0.2152 & 0.1394 & 0.1690 \\
\hline
\end{tabular}

Table 2. Neural Network Output Error

\begin{tabular}{cccccccc}
\hline $\begin{array}{c}\text { The number } \\
\text { of neurons }\end{array}$ & 7 & 8 & 9 & $\mathbf{1 0}$ & 11 & 12 & 13 \\
\hline $\begin{array}{c}\text { The error of } \\
\text { the network }\end{array}$ & 0.1217 & 0.1061 & 0.1141 & $\mathbf{0 . 1 0 0 5}$ & 0.1242 & 0.1320 & 0.1151 \\
\hline
\end{tabular}

Table 2. Neural Network Training Steps

\begin{tabular}{lccccccc}
\hline $\begin{array}{l}\text { The number } \\
\text { of neurons }\end{array}$ & 7 & 8 & 9 & $\mathbf{1 0}$ & 11 & 12 & 13 \\
\hline $\begin{array}{l}\text { The number } \\
\text { of training } \\
\text { steps }\end{array}$ & 122 & 120 & 119 & $\mathbf{1 0 8}$ & 110 & 120 & 114 \\
\hline
\end{tabular}

\section{Conclusion}

A kind of the AE signal recognition method is proposed based on wavelet transform and RBF neural network. The wavelet analysis is used to extract the energy of the detail characteristics of $\mathrm{AE}$ signal as the $\mathrm{AE}$ characteristics. $\mathrm{RBF}$ neural network is designed by the experiments and the classification experiments is conducted to the AE signal of the corrosion, crack and acoustic, and verify the superiority of the RBF network 
identification. This method proposed in this study can rapidly and accurately identify corrosion signal and interference signal. It has a reference value for the quantitative analysis to the corrosion situation of oil storage tanks.

\section{References}

[1] M. Y. Li, Z. D. Shang and H. C. Cai, "Acoustic emission detection and signal processing", Science Press, Beijing, (2011).

[2] T. Zhang and Y. B. Li, "The research and application of acoustic emission technology in the tank bottom corrosion detection, Sensor technology journal, vol. 23, no. 7, (2011), pp. 1050-1052.

[3] J. Lim and K. Micro, "Cracking in stainless steel pipe detection by using acoustic emission and crest factor technique", Instrumentation and Measurement Technology Conference, (2007) May 1-3, Warsaw, Poland.

[4] Y. H. Ma, K. Liu and D. Z. Yang, "The characteristics of acoustic emission signals recognition based on a typical BP network metal specimen tensile", Journal of Sichuan University (Engineering Science Edition), vol. 43, no. 2, (2011), pp. 252-255.

[5] H. Suzuki and T. Kinjo, "Fracture-mode determination of glass-fiber composites by various AE Processing", Progress in Acoustic Emission VIII, The Japanese Society for NDI, (1996).

[6] Q. Gang, B. Alan and H. Javad, "The wavelet transform in the acoustic emission signal feature extraction of the rubbing fault", 1st International Workshop on Database Technology and Applications, (2009), Wuhan, China.

[7] Y. Cui, X. L. Li and H. X. Peng, "The interface characterization of AE wavelet analysis of discontinuous reinforced based on metal matrix composites", Science Bulletin, vol. 43, no. 6, (2010), pp. 656-657.

[8] X. D Chang and L. X. Zhao, "Application of neural network on the ground of tank bottom AE leakage and corrosion signals differences", Overseas Oilfield Engineering, vol. 26, no. 7, (2010), pp. 48-50.

[9] H. K. Simon, "The principle of neural network", Mechanical Industry Press, Beijing, (2000).

[10] P. Zhang, K. Shi and R. S. Geng, "Application of wavelet transform in acoustic emission detection", Nondestructive testing, vol. 24, no. 10, (2002), pp. 436-439, 442.

[11] Tadej and Kosel, "Artificial neural network a tool for predicting failure strength of composite tensile coupons using acoustic emission technique", International Journal of Advanced Manufacturing Technology, vol. 44, no. 3, (2009), pp. 30-34.

[12] Y. X. Zhao and Y. G. Xu, "The fault pattern recognition of rolling bearing acoustic emission technology based on harmonic wavelet packet and BP neural network", Journal of vibration and shock, vol. 29, no. 10, (2010), pp. 163-166.

[13] Y. Q. Zhang, W. F Ni and W. Tan, "Analysis of acoustic emission signal characteristics of simulation based on wavelet transform", nondestructive testing, vol. 33, no. 1, (2011), pp. 16-17.

[14] L. Q. Han, "Artificial neural network tutorial”, Beijing University of Posts and Telecommunications press, Beijing, (2010).

[15] J. G. Yang, "Wavelet analysis and its application in engineering", Mechanical Industry Press, Beijing, (2007).

[16] Z. J. Cui, X. M. Shi and Y. L. Li, "The corrosion and protection of the oil and gas storage and transportation facilities", Petroleum Industry Press, Beijing, (2012).

[17] J. Zhou, L. C. Liu and J. P. Yang, "The denoising acoustic emission based on K-clustering and wavelet analysis", Journal of Petrochemical Universities, vol. 26, no. 3, (2013), pp. 16-19.

[18] J. R. Zhao, "The study of the identification method of acoustic emission signal processing system and source", Jilin University, (2010).

\section{Authors}

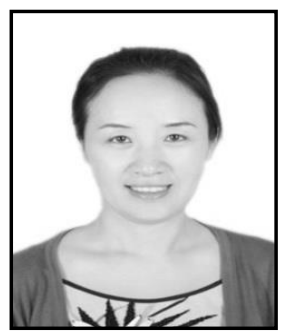

Shaohui Ma, received her Master Degree from Xi'an Jiaotong University in 2003. She is now an associate professor of Henan Mechanical and Electrical Engineering College in China. Her research interests include computer application, grid computing. She has published more than 7 papers on journals and conferences. 


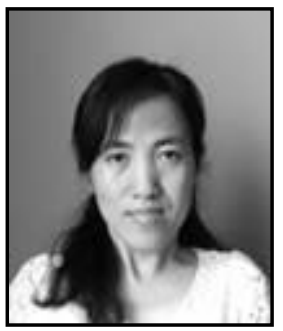

Xiangqian Chen, received her Master Degree from Henan University of Science and Technology in 2010.She is now an associate professor of Henan Mechanic and Electrical Engineering College in China. Her research interests include computer application; grid computing. She has published more than 6 papers on journals and conferences 
International Journal of Grid Distribution Computing Vol.8, No.2 (2015) 